YNU-HEPTh-99-102

KUCP-144

November 1999

Revised December 1999

\title{
Polarized Parton Distributions in the Photon and Factorization Scheme Dependence
}

\author{
KEn SASAKI* AND Tsuneo UEMATSU ${ }^{\dagger}$ \\ *Dept. of Physics, Faculty of Engineering, Yokohama National University \\ Yokohama 240-8501, JAPAN \\ ${ }^{\dagger}$ Dept. of Fundamental Sciences, FIHS, Kyoto University \\ Kyoto 606-8501, JAPAN
}

\begin{abstract}
Spin-dependent parton distributions in the polarized virtual photon are investigated in QCD up to the next-to-leading order (NLO). In the case $\Lambda^{2} \ll P^{2} \ll Q^{2}$, where $-Q^{2}\left(-P^{2}\right)$ is the mass squared of the probe (target) photon, parton distributions can be predicted completely up to the NLO, but they are factorization-scheme-dependent. Parton distributions are analyzed in four different factorization schemes and their scheme dependence are discussed. Particular attentions are paid to the axial anomaly effect on the first moments of quark parton distributions, and also to the large- $x$ behaviors of the parton distributions. Gluon distribution in the virtual photon is found to be factorization-scheme independent up to the NLO.
\end{abstract}

*e-mail address: sasaki@ed.ynu.ac.jp

${ }^{\dagger}$ e-mail address: uematsu@phys.h.kyoto-u.ac.jp 
In the past few years, the accuracy of the experimental data on the spin dependent structure function $g_{1}$ of the nucleon has been significantly improved [1]. Using these experimental data together with the already existing world data, it is now possible to extract polarized parton (i.e., quark and gluon) distributions in the nucleon in the framework of perturbative QCD. In fact several groups [2, 3, 4, 5] have carried out the next-to-leading order (NLO) QCD analysis on the polarized parton distributions in the nucleon recently. These parton distributions may be used for predicting the behaviors of other processes such as polarized Drell-Yan reactions and polarized semi-inclusive deep inelastic scatterings, and etc. The first moments of polarized parton distributions are particularly interesting due to their relevance for the spin structure of the nucleon [6, 0], where the axial anomaly plays an important role [8]. However, at the NLO and beyond in perturbative QCD, parton distribution functions become dependent on the factorization (or renormalization) scheme employed.

Recently, the first moment of the real photon structure function $g_{1}^{\gamma}$ has attracted attention in the literature [9, 10, 11]. More recently, the present authors investigated the spin-dependent structure function $g_{1}^{\gamma}\left(x, Q^{2}, P^{2}\right)$ of the virtual photon? in the NLO in QCD 14. The advantage in studying the virtual photon target is that, in the case $\Lambda^{2} \ll P^{2} \ll Q^{2}$, where $-Q^{2}\left(-P^{2}\right)$ is the mass squared of the probe (target) photon, and $\Lambda$ is the QCD scale parameter, we can calculate the whole structure function up to the NLO by the perturbative method [15, 16], in contrast to the case of the real photon target where there exist non-perturbative pieces in the NLO.

In this paper we analyze the polarized parton distributions in the virtual photon target. The behaviors of the parton distributions can be predicted entirely up to the NLO, but, of course, they are factorization-scheme-dependent. We carry out our analysis in four different factorization schemes, (i) $\overline{\mathrm{MS}}$, (ii) CI (chirally invariant) (it is also called as JET) [17, 18], (iii) AB (Adler-Bardeen) [19], and (iv) OS (offshell) [19], and see how the parton distributions change in each scheme. In particular, we examine in detail the axial anomaly effect on the first moments and the large$x$ behaviors of the parton distributions in each scheme. Gluon distribution in the virtual photon is found to be factorization-scheme independent up to the NLO.

\footnotetext{
1 The NLO QCD analysis on $g_{1}^{\gamma}$ for the real photon target was made by Stratmann and Vogelsang [12]. The leading order QCD correction to $g_{1}^{\gamma}$ was first studied by one of the authors [13].
} 
Let $\Delta q_{S}^{\gamma}\left(x, Q^{2}, P^{2}\right), \Delta q_{N S}^{\gamma}\left(x, Q^{2}, P^{2}\right), \Delta G^{\gamma}\left(x, Q^{2}, P^{2}\right), \Delta \Gamma^{\gamma}\left(x, Q^{2}, P^{2}\right)$ be the flavor singlet-, non-singlet-quark, gluon, and photon distribution functions, respectively, in the longitudinally polarized virtual photon with mass $-P^{2}$. In the leading order of the electromagnetic coupling constant, $\alpha=e^{2} / 4 \pi, \Delta \Gamma^{\gamma}$ does not evolve with $Q^{2}$ and is set to be $\Delta \Gamma^{\gamma}\left(x, Q^{2}, P^{2}\right)=\delta(1-x)$. In terms of these parton distribution functions, the polarized virtual photon structure function $g_{1}^{\gamma}\left(x, Q^{2}, P^{2}\right)$ is expressed in the QCD improved parton model as [14]

$$
\begin{array}{r}
g_{1}^{\gamma}\left(x, Q^{2}, P^{2}\right)=\int_{x}^{1} \frac{d y}{y}\left\{\Delta q_{S}^{\gamma}\left(y, Q^{2}, P^{2}\right) \Delta C_{S}^{\gamma}\left(\frac{x}{y}, Q^{2}\right)+\Delta G^{\gamma}\left(y, Q^{2}, P^{2}\right) \Delta C_{G}^{\gamma}\left(\frac{x}{y}, Q^{2}\right)\right. \\
\left.+\Delta q_{N S}^{\gamma}\left(y, Q^{2}, P^{2}\right) \Delta C_{N S}^{\gamma}\left(\frac{x}{y}, Q^{2}\right)\right\}+\Delta C_{\gamma}^{\gamma}\left(x, Q^{2}\right)
\end{array}
$$

where $\Delta C_{S}^{\gamma}\left(\Delta C_{N S}^{\gamma}\right), \Delta C_{G}^{\gamma}$, and $\Delta C_{\gamma}^{\gamma}$ are the coefficient functions corresponding to singlet(non-singlet)-quark, gluon, and photon, respectively, and are independent of $P^{2}$. The Mellin moments of $g_{1}^{\gamma}$ is written as

$$
g_{1}^{\gamma}\left(n, Q^{2}, P^{2}\right)=\Delta \boldsymbol{C}^{\gamma}\left(n, Q^{2}\right) \cdot \Delta \boldsymbol{q}^{\gamma}\left(n, Q^{2}, P^{2}\right)
$$

where

$$
\begin{aligned}
\Delta \boldsymbol{C}^{\gamma}\left(n, Q^{2}\right) & =\left(\Delta C_{S}^{\gamma}, \Delta C_{G}^{\gamma}, \Delta C_{N S}^{\gamma}, \Delta C_{\gamma}^{\gamma}\right) \\
\Delta \boldsymbol{q}^{\gamma}\left(n, Q^{2}, P^{2}\right) & =\left(\Delta q_{S}^{\gamma}, \Delta G^{\gamma}, \Delta q_{N S}^{\gamma}, \Delta \Gamma^{\gamma}\right)
\end{aligned}
$$

and the matrix notation is implicit.

The parton distribution $\Delta \boldsymbol{q}^{\gamma}$ satisfies the inhomogeneous evolution equation [20, 21, 22, 23]. The explicit expressions of $\Delta q_{S}^{\gamma}, \Delta G^{\gamma}$, and $\Delta q_{N S}^{\gamma}$ up to the NLO are easily derived from Eq.(4.46) of Ref.[14]. They are given[] in terms of one-(two-) loop hadronic anomalous dimensions $\Delta \gamma_{i j}^{(0), n}\left(\Delta \gamma_{i j}^{(1), n}\right)(i, j=\psi, G)$ and $\Delta \gamma_{N S}^{(0), n}\left(\Delta \gamma_{N S}^{(1), n}\right)$, one-(two-) loop anomalous dimensions $\Delta K_{i}^{(0), n}\left(\Delta K_{i}^{(1), n}\right)(i=\psi, G, N S)$ which represent the mixing between photon and three hadronic operators $R_{i}^{n}(i=\psi, G, N S)$, and finally $A_{i}^{n}$, the one-loop photon matrix elements of hadronic operators renormalized at $\mu^{2}=P^{2}\left(=-p^{2}\right)$,

$$
\left.\left\langle\gamma(p)\left|R_{i}^{n}(\mu)\right| \gamma(p)\right\rangle\right|_{\mu^{2}=P^{2}}=\frac{\alpha}{4 \pi} A_{i}^{n} \quad(i=\psi, G, N S) .
$$

\footnotetext{
${ }^{2}$ We use the same notations as in Ref. 14], except that the symbol $\Delta$ has been appended to all the spin-dependent anomalous dimensions and coefficient functions.
} 
Although $g_{1}^{\gamma}$ is a physical quantity and thus unique, there remains a freedom in the factorization of $g_{1}^{\gamma}$ into $\Delta \boldsymbol{C}^{\gamma}$ and $\Delta \boldsymbol{q}^{\gamma}$. Given the formula Eq.(2), we can always redefine $\Delta \boldsymbol{C}^{\gamma}$ and $\Delta \boldsymbol{q}^{\gamma}$ as follows [20]:

$$
\begin{aligned}
\left.\Delta \boldsymbol{C}^{\gamma}\left(n, Q^{2}\right) \rightarrow \Delta \boldsymbol{C}^{\gamma}\left(n, Q^{2}\right)\right|_{a} & \equiv \Delta \boldsymbol{C}^{\gamma}\left(n, Q^{2}\right) Z_{a}^{-1}\left(n, Q^{2}\right) \\
\left.\Delta \boldsymbol{q}^{\gamma}\left(n, Q^{2}, P^{2}\right) \rightarrow \Delta \boldsymbol{q}\left(n, Q^{2}, P^{2}\right)\right|_{a} & \equiv Z_{a}\left(n, Q^{2}\right) \Delta \boldsymbol{q}^{\gamma}\left(n, Q^{2}, P^{2}\right)
\end{aligned}
$$

where $\left.\Delta \boldsymbol{C}^{\gamma}\right|_{a}$ and $\left.\boldsymbol{\Delta} \boldsymbol{q}\right|_{a}$ correspond to the quantities in a new factorization scheme- $a$. Note that the coefficient functions and anomalous dimensions are closely connected under factorization. We will study the factorization scheme dependence of parton distribution up to the NLO, by which we mean that a scheme transformation for the coefficient functions is considered up to the one-loop order, since a NLO prediction for $g_{1}^{\gamma}$ is given by the one-loop coefficient functions and anomalous dimensions up to the two-loop order.

The most general form of a transformation for the coefficient functions in oneloop order, from $\overline{\mathrm{MS}}$ scheme to a new factorization scheme- $a$, is given by

$$
\begin{aligned}
& \Delta C_{S, a}^{\gamma, n}=\Delta C_{S, \frac{n}{\mathrm{MS}}}^{\gamma, n}-<e^{2}>\frac{\alpha_{s}}{2 \pi} w_{S}(n, a) \\
& \Delta C_{G, a}^{\gamma, n}=\Delta C_{G, \overline{\mathrm{MS}}}^{\gamma, n}-<e^{2}>\frac{\alpha_{S}}{2 \pi} z(n, a) \\
& \Delta C_{N S, a}^{\gamma, n}=\Delta C_{N S, \overline{\mathrm{MS}}}^{\gamma, n}-\frac{\alpha_{s}}{2 \pi} w_{N S}(n, a) \\
& \Delta C_{\gamma, a}^{\gamma, n}=\Delta C_{\gamma, \frac{\mathrm{MS}}{\gamma, n}}^{\gamma}-\frac{\alpha}{\pi} 3<e^{4}>\hat{z}(n, a)
\end{aligned}
$$

where $<e^{2}>=\sum_{i} e_{i}^{2} / N_{f},<e^{4}>=\sum_{i} e_{i}^{4} / N_{f}$, with $N_{f}$ being the number of flavors of active quarks and $e_{i}$ being the electric charge of $i$-flavor-quark. The $z(n, a)$ $(\hat{z}(n, a))$ term tells how much of the QCD (QED) axial-anomaly effect is transferred to the coefficient function in the new factorization scheme. Note that $\Delta C_{\gamma}^{\gamma, n} \frac{n}{\mathrm{MS}}$ has been obtained from $\Delta C_{G}^{\gamma,}, \overline{\mathrm{MS}}$, with changes: $\alpha_{S} / 2 \pi \rightarrow\left(2 \alpha / \alpha_{S}\right) \times\left(\alpha_{S} / 2 \pi\right)$, $<e^{2}>\rightarrow 3<e^{4}>$, and 3 is the number of colors. Once the relations (5) between the coefficient functions in the $a$-scheme and $\overline{\mathrm{MS}}$ scheme are given, we can derive corresponding transformation rules from $\overline{\mathrm{MS}}$ scheme to $a$-scheme for the relevant two-loop anomalous dimensins. We find ${ }^{3}$

$$
\Delta \gamma_{\psi \psi, a}^{(1), n}=\Delta \gamma_{\psi \psi, \overline{\mathrm{MS}}}^{(1), n}+2 z(n, a) \Delta \gamma_{G \psi}^{(0), n}+4 \beta_{0} w_{S}(n, a)
$$

\footnotetext{
${ }^{3}$ For detailed derivation of the transformation rules, see Ref. 24.
} 


$$
\begin{aligned}
\Delta \gamma_{\psi G, a}^{(1), n}= & \Delta \gamma_{\psi G, \overline{\mathrm{MS}}}^{(1), n}+2 z(n, a)\left[\Delta \gamma_{G G}^{(0), n}-\Delta \gamma_{\psi \psi}^{(0), n}+2 \beta_{0}\right] \\
& +2 w_{S}(n, a) \Delta \gamma_{\psi G}^{(0), n} \\
\Delta \gamma_{G \psi, a}^{(1), n}= & \Delta \gamma_{G \psi, \overline{\mathrm{MS}}}^{(1), n}-2 w_{S}(n, a) \Delta \gamma_{G \psi}^{(0), n} \\
\Delta \gamma_{G G, a}^{(1), n}= & \Delta \gamma_{G G, \overline{\mathrm{MS}}}^{(1), n}-2 z(n, a) \Delta \gamma_{G \psi}^{(0), n} \\
\Delta \gamma_{N S, a}^{(1), n}= & \Delta \gamma_{N S, \overline{\mathrm{MS}}}^{(1), n}+4 \beta_{0} w_{N S}(n, a) \\
\Delta K_{S, a}^{(1), n}= & \Delta K_{S, \overline{\mathrm{MS}}}^{(1), n}+2 w_{S}(n, a) \Delta K_{S}^{(0), n}+4 \hat{z}(n, a) 3<e^{2}>\Delta \gamma_{\psi \psi}^{(0), n} \\
\Delta K_{G, a}^{(1), n}= & \Delta K_{G, \overline{\mathrm{MS}}}^{(1), n}+4 \hat{z}(n, a) 3<e^{2}>\Delta \gamma_{G \psi}^{(0), n} \\
\Delta K_{N S, a}^{(1), n}= & \Delta K_{N S, \overline{\mathrm{MS}}}^{(1), n}+2 w_{N S}(n, a) \Delta K_{N S}^{(0), n} \\
& +4 \hat{z}(n, a) 3\left(<e^{4}>-<e^{2}>^{2}\right) \Delta \gamma_{N S}^{(0), n}
\end{aligned}
$$

where $\beta_{0}=11-\frac{2}{3} N_{f}$ is the one-loop coefficient of the QCD beta function.

Since the one-loop photon matrix elements of the hadronic operators, $A_{\psi}^{n}$ and $A_{N S}^{n}$ in Eq.(3), are related to each other as $A_{N S}^{n}=A_{\psi}^{n}\left(<e^{4}>-<e^{2}>^{2}\right) /<e^{2}>$ and the sum $\left(\Delta C_{\gamma}^{\gamma, n} / \frac{\alpha}{4 \pi}+<e^{2}>A_{\psi}^{n}+A_{N S}^{n}\right)$ is factorization-scheme-independent in one-loop order 14, we find from Eq.(5)

$$
\begin{aligned}
A_{\psi, a}^{n} & =A_{\psi, \overline{\mathrm{MS}}}^{n}+12<e^{2}>\hat{z}(n, a) \\
A_{G, a}^{n} & =A_{G, \overline{\mathrm{MS}}}^{n}=0 \\
A_{N S, a}^{n} & =A_{N S, \overline{\mathrm{MS}}}^{n}+12\left(<e^{4}>-<e^{2}>^{2}\right) \hat{z}(n, a) .
\end{aligned}
$$

Note that $A_{G}^{n}=0$ in one-loop order.

It is possible to choose $z(n, a)$ and $\hat{z}(n, a)$ arbitrarily. But, here, we are interested in the QCD and QED anomaly effects on the parton distributions in the virtual photon, and both QCD and QED anomalies originate from the similar triangle diagrams. Therefore, we take in the following $z(n, a)=\hat{z}(n, a)$. With this choice, the relation between the one-loop gluon and photon coefficient functions

$$
\Delta B_{\gamma}^{n}=\frac{2}{N_{f}} \Delta B_{G}^{n},
$$

holds not only in the $\overline{\mathrm{MS}}$ scheme but also in the $a$-scheme, where $\Delta B_{\gamma}^{n}$ and $\Delta B_{G}^{n}$ are defined as

$$
\Delta C_{G}^{\gamma, n}=<e^{2}>\left(\frac{\alpha_{s}}{4 \pi} \Delta B_{G}^{n}+\mathcal{O}\left(\alpha_{s}^{2}\right)\right)
$$




$$
\Delta C_{\gamma}^{\gamma, n}=\frac{\alpha}{4 \pi} 3 N_{f}<e^{4}>\left(\Delta B_{\gamma}^{n}+\mathcal{O}\left(\alpha_{s}\right)\right)
$$

Also in one-loop order we have $w_{S}(n, a)=w_{N S}(n, a)$. Thus from now on, we set $\hat{z}(n, a)=z(n, a)$ and $w_{S}(n, a)=w_{N S}(n, a) \equiv w(n, a)$.

Now let us discuss the features of several factorization schemes.

(i) [The $\overline{\mathrm{MS}}$ scheme] This is the only scheme in which both relevant oneloop coefficient functions and two-loop anomalous dimensions were actually calculated [25, 7, 26, 27]. In fact there still remain ambuguities in the $\overline{\mathrm{MS}}$ scheme, depending on how to handle $\gamma_{5}$ in $n$ dimensions. The $\overline{\mathrm{MS}}$ scheme we call here is the one due to Mertig and van Neerven [26] and Vogelsang [27], in which the first moment of the non-singlet quark operator vanishes, corresponding to the conservation of the non-singlet axial current. Indeed we have $\Delta \gamma_{N S, \overline{\mathrm{MS}}}^{(1), n=1}=0$. Explicit expressions of the relevant one-loop coefficient functions and two-loop anomalous dimensions can be found, for example, in Appendix of Ref. [14. In the $\overline{\mathrm{MS}}$ scheme, the QCD (QED) axial anomaly resides in the quark distributions and not in the gluon (photon) coefficient function [7, 17]. In fact we observe

$$
\Delta \gamma_{\psi \psi, \overline{\mathrm{MS}}}^{(1), n=1}=24 C_{F} T_{f} \neq 0, \quad \Delta B_{G, \overline{\mathrm{MS}}}^{n=1}=\Delta B_{\gamma, \overline{\mathrm{MS}}}^{n=1}=0 .
$$

Also the first moment of the one-loop photon matrix element of quark operators gains the non-zero values, i.e.,

$$
A_{\psi, \overline{\mathrm{MS}}}^{n=1}=\frac{<e^{2}>}{<e^{4}>-<e^{2}>^{2}} A_{N S, \overline{\mathrm{MS}}}^{n=1}=-12<e^{2}>N_{f}
$$

which is due to the QED axial anomaly.

(ii) [The chirally invariant (CI) scheme] In this scheme the factorization of the photon-gluon (photon-photon) cross section into the hard and soft parts is made so that chiral symmetry is respected and all the anomaly effects are absorbed into the gluon (photon) coefficient function [17, 18]. Thus the spin-dependent quark distributions in the CI scheme are anomaly-free. In particular, we have

$$
\begin{array}{rlrl}
\Delta B_{G, \mathrm{CI}}^{n=1} & =-2 N_{f}, & & \Delta \gamma_{\psi \psi, \mathrm{CI}}^{(1), n=1}=0 \\
\Delta B_{\gamma, \mathrm{CI}}^{n=1}=-4, & & A_{\psi, \mathrm{CI}}^{n=1}=A_{N S, \mathrm{CI}}^{n=1}=0 .
\end{array}
$$

The transformation from $\overline{\mathrm{MS}}$ scheme to CI scheme is achieved by

$$
w(n, a=\mathrm{CI})=0, \quad z(n, a=\mathrm{CI})=2 N_{f} \frac{1}{n(n+1)} .
$$


It has been argued by Cheng [17] and Müller and Teryaev [18] that the $x$-dependence of the axial-anomaly effect is uniquely fixed and that its $x$-behavior leads to the transformation rule (14) and thus to the CI scheme.

(iii) [The Adler-Bardeen (AB) scheme] Ball, Forte and Ridolfi [19 proposed several CI-like schemes in which features of the CI scheme (CI-relations in Eq.(12)) are kept intact. One of them is the Adler-Bardeen (AB) scheme which was introduced by requiring that the change from the $\overline{\mathrm{MS}}$ scheme to this scheme be independent of $x$, so that the large and small $x$ behavior of the gluon (photon) coefficient function is unchanged. In moment space we have

$$
w(n, a=\mathrm{AB})=0, \quad z(n, a=\mathrm{AB})=N_{f} \frac{1}{n} .
$$

(iv) [The off-shell (OS) scheme] In this scheme [19] we renormalize operators while keeping the incoming particle off-shell, $p^{2} \neq 0$, so that at renormalization (factorization) point $\mu^{2}=-p^{2}$, the finite terms vanish. This is exactly the same as "the momentum subtraction scheme" which was used some time ago to calculate, for instance, the polarized quark and gluon coefficient functions [28, 8] The CIrelations in Eq.(12) hold in the OS scheme, since the axial anomaly appears as a finite term in the calculation of the triangle graph for $j_{5}^{\mu}$ between external gluons (photons) and the finite term is thrown away in this scheme. The transformation from the $\overline{\mathrm{MS}}$ scheme to the OS scheme is made by choosing

$$
\begin{array}{r}
w(n, a=\mathrm{OS})=C_{F}\left\{\left[S_{1}(n)\right]^{2}+3 S_{2}(n)-S_{1}(n)\left(\frac{1}{n}-\frac{1}{(n+1)}\right)\right. \\
\left.-\frac{7}{2}+\frac{2}{n}-\frac{3}{n+1}-\frac{1}{n^{2}}+\frac{2}{(n+1)^{2}}\right\} \\
z(n, a=\mathrm{OS})=N_{f}\left\{-\frac{n-1}{n(n+1)} S_{1}(n)+\frac{1}{n}+\frac{1}{n^{2}}-\frac{4}{(n+1)^{2}}\right\} .
\end{array}
$$

It is noted that in the OS scheme we have $A_{\psi, \text { OS }}^{n}=A_{N S \text {, OS }}^{n}=0$ for all $n$.

Now we examine the factoraization scheme dependence of the polarized parton distributions in virtual photon. The two-loop anomalous dimensions of the spindependent operators and one-loop photon matrix elements of the hadronic operators in the $\overline{\mathrm{MS}}$ scheme are already known. Corresponding quantities in a particular

\footnotetext{
${ }^{4}$ In fact, the author of Ref. 8 treated the $n=1$ moment of the gluon coefficient function differently from other moments [14.
} 
scheme are obtained through the transformation rules in Eq.(6). Using these quantities, we get the NLO predictions for the moments of polarized parton distributions in virtual photon in a particular factorization scheme. We find that the gluon distribution is factorization-scheme independent up to the NLO f,

$$
\left.\Delta G^{\gamma}\left(n, Q^{2}, P^{2}\right)\right|_{a}=\left.\Delta G^{\gamma}\left(n, Q^{2}, P^{2}\right)\right|_{\overline{\mathrm{MS}}},
$$

where $a$ means CI, AB, OS, or any other factorization scheme. This can be seen from the direct calculation or from the notion that, up to the NLO, $\left.\Delta G^{\gamma}\right|_{a}$ satisfies the same evolution equation as $\left.\Delta G^{\gamma}\right|_{\overline{\mathrm{MS}}}$ with the same initial condition at $Q^{2}=P^{2}$, namely, $\left.\Delta G^{\gamma}\left(n, P^{2}, P^{2}\right)\right|_{a}=\left.\Delta G^{\gamma}\left(n, P^{2}, P^{2}\right)\right|_{\overline{\mathrm{MS}}}=0$.

(1) [The first moments] For all three factorization schemes, $a=\mathrm{CI}, \mathrm{AB}$, OS, we have

$$
w(n=1, a)=0, \quad z(n=1, a)=N_{f} \quad \text { for } a=\mathrm{CI}, \mathrm{AB}, \mathrm{OS}
$$

These schemes, therefore, give the same first moments for the parton distributions. In fact, from Eqs.(17) and (11) we find $A_{\psi, a}^{n=1}=A_{N S, a}^{n=1}=0$. This leads to

$$
\left.\Delta q_{S}^{\gamma}\left(n=1, Q^{2}, P^{2}\right)\right|_{a}=\left.\Delta q_{N S}^{\gamma}\left(n=1, Q^{2}, P^{2}\right)\right|_{a}=0
$$

in the NLO for $a=\mathrm{CI}, \mathrm{AB}$, OS. In these schemes, the axial anomaly effects are transfered to the gluon and photon coefficient functions. On the other hand, in $\overline{\mathrm{MS}}$ scheme we obtain

$$
\begin{aligned}
\Delta q_{S}^{\gamma}(n & \left.=1, Q^{2}, P^{2}\right)\left.\right|_{\overline{\mathrm{MS}}}=\left[-\frac{\alpha}{\pi} 3<e^{2}>N_{f}\right]\left\{1-\frac{2}{\beta_{0}} \frac{\alpha_{s}\left(P^{2}\right)-\alpha_{s}\left(Q^{2}\right)}{\pi} N_{f}\right\} \\
\Delta q_{N S}^{\gamma}(n & \left.=1, Q^{2}, P^{2}\right)\left.\right|_{\overline{\mathrm{MS}}}=\left[-\frac{\alpha}{\pi} 3\left(<e^{4}>-<e^{2}>^{2}\right) N_{f}\right]\left\{1+\mathcal{O}\left(\alpha_{s}^{2}\right)\right\}
\end{aligned}
$$

For gluon distribution, we have

$$
\begin{aligned}
\left.\Delta G^{\gamma}\left(n=1, Q^{2}, P^{2}\right)\right|_{\overline{\mathrm{MS}}} & =\left.\Delta G^{\gamma}\left(n=1, Q^{2}, P^{2}\right)\right|_{a} \\
& =\frac{12 \alpha}{\pi \beta_{0}}<e^{2}>N_{f} \frac{\alpha_{s}\left(Q^{2}\right)-\alpha_{s}\left(P^{2}\right)}{\alpha_{s}\left(Q^{2}\right)}
\end{aligned}
$$

\footnotetext{
${ }^{5} \mathrm{By}$ NLO we mean that we consider a general scheme transformation for the coefficient functions up to the one-loop order, which is given by Eq.(5).

${ }^{6}$ The detailed derivation will be reported elsewhere 24.
} 
The polarized structure function $g_{1}^{\gamma}\left(x, Q^{2}, P^{2}\right)$ of the virtual photon satisfies the following sum rule 14, 29]:

$$
\begin{aligned}
\int_{0}^{1} d x g_{1}^{\gamma}\left(x, Q^{2}, P^{2}\right) & =-\frac{3 \alpha}{\pi}<e^{4}>N_{f}\left(1-\frac{\alpha_{s}\left(Q^{2}\right)}{\pi}\right) \\
& +\frac{6 \alpha}{\pi \beta_{0}}\left[<e^{2}>N_{f}\right]^{2} \frac{\alpha_{s}\left(P^{2}\right)-\alpha_{s}\left(Q^{2}\right)}{\pi}+\mathcal{O}\left(\alpha_{s}^{2}\right) .
\end{aligned}
$$

This sum rule is of course the factorization-scheme independent. Now we examine how the scheme-dependent parton distributions contribute to this sum rule. In the CI-like schemes ( $a=\mathrm{CI}, \mathrm{AB}, \mathrm{OS})$, the first moment of the quark distributions vanish in the NLO, and thus the contribution to the sum rule come from the gluon and photon distributions. Since

$$
\begin{aligned}
& \Delta C_{G, a}^{\gamma, n=1}=-<e^{2}>\frac{\alpha_{s}\left(Q^{2}\right)}{2 \pi} N_{f} \\
& \Delta C_{\gamma, a}^{\gamma, n=1}=-\frac{3 \alpha}{\pi}<e^{4}>N_{f}\left(1-\frac{\alpha_{s}\left(Q^{2}\right)}{\pi}\right)
\end{aligned}
$$

we see that $\left[\left.\Delta C_{G, a}^{\gamma, n=1} \Delta G^{\gamma}\left(n=1, Q^{2}, P^{2}\right)\right|_{a}+\Delta C_{\gamma, a}^{\gamma, n=1}\right]$ leads to the result (22). On the other hand, in the $\overline{\mathrm{MS}}$ scheme, the one-loop gluon and photon coefficient functions vanish, $\Delta B_{G, \overline{\mathrm{MS}}}^{n=1}=\Delta B_{\gamma, \overline{\mathrm{MS}}}^{n=1}=0$ and, therefore, the sum rule is derived from the quark contributions. Indeed we have in one-loop order

$$
\frac{1}{<e^{2}>} \Delta C_{S, \frac{n=1}{\mathrm{MS}}}^{\gamma, n}=\Delta C_{N S, \overline{\mathrm{MS}}}^{\gamma, n=1}=\left(1-\frac{\alpha_{s}\left(Q^{2}\right)}{\pi}\right)
$$

and find that

$$
\left.\Delta C_{S, \frac{n=1}{\mathrm{MS}}}^{\gamma} \Delta q_{S}^{\gamma}\left(n=1, Q^{2}, P^{2}\right)\right|_{\overline{\mathrm{MS}}}+\left.\Delta C_{N S, \overline{\mathrm{MS}}}^{\gamma, n=1} \Delta q_{N S}^{\gamma}\left(n=1, Q^{2}, P^{2}\right)\right|_{\overline{\mathrm{MS}}}
$$

leads to the same result.

It is interesting to note that the sum rule (22) is the consequence of the axial anomaly and that in the CI-like schemes the anomaly effect resides in the gluon contribution while, in the $\overline{\mathrm{MS}}$, in the quark contributions. Furthermore, the first term of the sum rule (22) is coming from the QED axial anomaly and the second is from the QCD axial anomaly.

(2) [behaviors near $x=1$ ] The behaviors of parton distributions near $x=1$ are governed by the large- $n$ limit of those moments. In the leading order (LO), parton 
distributions are factorization-scheme independent. For large $n,\left.\Delta q_{S}^{\gamma}\left(n, Q^{2}, P^{2}\right)\right|_{\text {LO }}$ and $\left.\Delta q_{N S}^{\gamma}\left(n, Q^{2}, P^{2}\right)\right|_{\text {LO }}$ behave as $1 /(n \ln n)$, while $\left.\Delta G^{\gamma}\left(n, Q^{2}, P^{2}\right)\right|_{\text {LO }} \propto 1 /(n \ln n)^{2}$. Thus in $x$ space, the parton distributions vanish for $x \rightarrow 1$. In fact we find

$$
\begin{aligned}
\left.\Delta q_{S}^{\gamma}\left(x, Q^{2}, P^{2}\right)\right|_{\mathrm{LO}} & \approx \frac{\alpha}{4 \pi} \frac{4 \pi}{\alpha_{s}\left(Q^{2}\right)} N_{f}<e^{2}>\frac{9}{4} \frac{-1}{\ln (1-x)} \\
\left.\Delta G^{\gamma}\left(x, Q^{2}, P^{2}\right)\right|_{\mathrm{LO}} & \approx \frac{\alpha}{4 \pi} \frac{4 \pi}{\alpha_{s}\left(Q^{2}\right)} N_{f}<e^{2}>\frac{1}{2} \frac{-\ln x}{\ln ^{2}(1-x)} .
\end{aligned}
$$

The behaviors of $\Delta q_{N S}^{\gamma}\left(x, Q^{2}, P^{2}\right)$ for $x \rightarrow 1$, both in the LO and NLO, are always given by the corresponding expressions for $\Delta q_{S}^{\gamma}\left(x, Q^{2}, P^{2}\right)$ with the replacement of the charge factor $<e^{2}>$ with $\left(<e^{4}>-<e^{2}>^{2}\right)$.

In the $\overline{\mathrm{MS}}$ scheme, the moment $\left.\Delta q_{S}^{\gamma}\left(n, Q^{2}, P^{2}\right)\right|_{\mathrm{NLO}}, \overline{\mathrm{MS}}$ behaves as $(\ln n) / n$ in large $n$ limit, while $\left.\Delta G^{\gamma}\left(n, Q^{2}, P^{2}\right)\right|_{\mathrm{NLO}}, \overline{\mathrm{MS}} \propto 1 / n^{2}$. Thus we have near $x=1$

$$
\begin{aligned}
\left.\Delta q_{S}^{\gamma}\left(x, Q^{2}, P^{2}\right)\right|_{\mathrm{NLO}, \overline{\mathrm{MS}}} & \approx \frac{\alpha}{4 \pi} N_{f}<e^{2}>6[-\ln (1-x)] \\
\left.\Delta G^{\gamma}\left(x, Q^{2}, P^{2}\right)\right|_{\mathrm{NLO}, \overline{\mathrm{MS}}} & \approx \frac{\alpha}{4 \pi} N_{f}<e^{2}>3[-\ln x] .
\end{aligned}
$$

It is remarkable that, in the $\overline{\mathrm{MS}}$ scheme, quark parton distributions, $\left.\Delta q_{S}^{\gamma}(x)\right|_{\mathrm{NLO}}, \overline{\mathrm{MS}}$ and $\left.\Delta q_{N S}^{\gamma}(x)\right|_{\mathrm{NLO}} \overline{\mathrm{MS}}$ diverge as $[-\ln (1-x)]$ for $x \rightarrow 1$. Recall that $\left.\Delta G^{\gamma}\right|_{\mathrm{NLO}}$ is scheme-independent. The NLO quark distributions in the CI and AB schemes also diverge as $x \rightarrow 1$. In fact we obtain for large $x$

$$
\begin{aligned}
\left.\Delta q_{S}^{\gamma}\left(x, Q^{2}, P^{2}\right)\right|_{\mathrm{NLO}, \mathrm{CI}} & \approx \frac{\alpha}{4 \pi} N_{f}<e^{2}>6[-\ln (1-x)] \\
\left.\Delta q_{S}^{\gamma}\left(x, Q^{2}, P^{2}\right)\right|_{\mathrm{NLO}, \mathrm{AB}} & \approx \frac{\alpha}{4 \pi} N_{f}<e^{2}>6[-\ln (1-x)+2] .
\end{aligned}
$$

On the other hand, the OS scheme gives quite different behaviors near $x=1$ for the quark distributions. We find that the moment $\left.\Delta q_{S}^{\gamma}\left(n, Q^{2}, P^{2}\right)\right|_{\mathrm{NLO}}$ in the OS scheme behaves as $1 / n$ for large $n$. Thus, in $x$ space, $\left.\Delta q_{S}^{\gamma}\left(x, Q^{2}, P^{2}\right)\right|_{\mathrm{NLO} \text {, OS }}$ does not diverge for $x \rightarrow 1$ and approaches a constant value:

$$
\left.\Delta q_{S}^{\gamma}\left(x, Q^{2}, P^{2}\right)\right|_{\mathrm{NLO}, \text { OS }} \longrightarrow \frac{\alpha}{4 \pi} N_{f}<e^{2}>\left[\frac{69}{8}+\frac{3}{4} N_{f}\right] .
$$

We can show 24] that, as $x \rightarrow 1$, the polarized virtual photon structure function $g_{1}^{\gamma}\left(x, Q^{2}, P^{2}\right)$ in the NLO approaches a constant value

$$
\kappa=\frac{\alpha}{4 \pi} N_{f}<e^{4}>\left[-\frac{51}{8}+\frac{3}{4} N_{f}\right]
$$


and the result is factorization-scheme independent. In the $\overline{\mathrm{MS}}, \mathrm{CI}, \mathrm{AB}$ schemes, the NLO quark parton distributions and hence their contributions to $g_{1}^{\gamma}\left(x, Q^{2}, P^{2}\right)$ diverge as $[-\ln (1-x)]$ for $x \rightarrow 1$. However, the one-loop photon coefficient function $\Delta C_{\gamma}^{\gamma}(x)$ in these schemes also diverges as $[-\ln (1-x)]$ with the opposite sign and the sum becomes finite. On the other hand, in the OS scheme, both the quark distributions and photon coefficient function remain finite as $x \rightarrow 1$. Therefore, as far as the large $x$-behaviors of quark parton distributions and photon (also gluon) coefficient functions are concerned, the OS scheme is more appropriate than other schemes in the sence that they remain finite.

The constant value $\kappa$ in Eq.(31) is negative] unless $N_{f} \geq 9$. Consequently, it seems superficially that QCD with 8 flavors or less predicts that the structure function $g_{1}^{\gamma}\left(x, Q^{2}, P^{2}\right)$ turns out to be negative for $x$ very close to 1 , since the leading

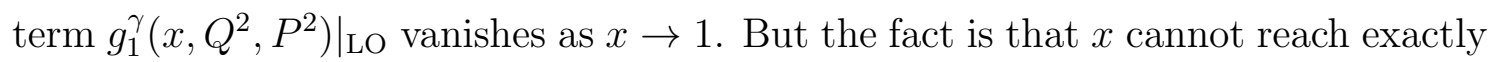
one. The constraint $(p+q)^{2} \geq 0$ gives $x \leq x_{\max }=\frac{Q^{2}}{Q^{2}+P^{2}}$, and we find

$$
\left.g_{1}^{\gamma}\left(x=x_{\max }, Q^{2}, P^{2}\right)\right|_{\mathrm{LO}}>\frac{\alpha}{4 \pi} N_{f}<e^{4}>\beta_{0}
$$

and the sum $\left.g_{1}^{\gamma}\left(x=x_{\max }, Q^{2}, P^{2}\right)\right|_{\mathrm{LO}+\mathrm{NLO}}$ is indeed positive.

(3) [Numerical analysis] The parton distribution functions are recovered from the moments by the inverse Mellin transformation. In Fig. 1 we plot the factorization scheme dependence of the singlet quark distribution $\Delta q_{S}^{\gamma}\left(x, Q^{2}, P^{2}\right)$ beyond the LO in units of $\left(3 N_{f}<e^{2}>\alpha / \pi\right) \ln \left(Q^{2} / P^{2}\right)$. We have taken $N_{f}=3, Q^{2}=30 \mathrm{GeV}^{2}$, $P^{2}=1 \mathrm{GeV}^{2}$, and the QCD scale parameter $\Lambda=0.2 \mathrm{GeV}$. All three CI-like (i.e., $\mathrm{CI}, \mathrm{AB}$ and $\mathrm{OS}$ ) lines cross the $x$-axis nearly at the same point, just below $x=0.5$, while the $\overline{\mathrm{MS}}$ line crosses at above $x=0.5$. This is understandable since we saw from Eqs.(19, 20) that the first moment of $\Delta q_{S}^{\gamma}$ vanishes in the CI-like schemes while it is negative in the $\overline{\mathrm{MS}}$ scheme. As $x \rightarrow 1$, we observe that the $\overline{\mathrm{MS}}, \mathrm{CI}$, and $\mathrm{AB}$ lines continue to increase while the OS line starts to drop. We also see that the $\overline{\mathrm{MS}}$ and CI lines tend to merge and the AB line comes above those two lines. These behaviors are inferred from Eqs.(27-29, 30).

Fig. 2 shows the $Q^{2}$-dependence of $\Delta q_{S}^{\gamma}\left(x, Q^{2}, P^{2}\right)$ in the OS scheme in units of $\left(3 N_{f}<e^{2}>\alpha / \pi\right) \ln \left(Q^{2} / P^{2}\right)$. Three lines with $Q^{2}=30,50$ and $100 \mathrm{GeV}^{2}$

${ }^{7}$ The constant value $\kappa$ coincides exactly with the one given in Eq.(4.39) of Ref. 30], which was derived as the large $n$ limit of the moment of the NLO term $b_{2}(x)$ for the unpolarized structure function $F_{2}^{\gamma} 31$. 
almost overlap in whole $x$ region except in the vicinity of $x=1$. Indeed we see from Fig. 2 that in the OS scheme $\Delta q_{S}^{\gamma}$ beyond the LO behaves approximately as the one obtained from the box (tree) diagram calculation,

$$
\Delta q_{S}^{\gamma(\text { Box })}\left(x, Q^{2}, P^{2}\right)=(2 x-1) 3 N_{f}<e^{2}>\frac{\alpha}{\pi} \ln \frac{Q^{2}}{P^{2}}
$$

Concerning the non-singlet quark distribution $\Delta q_{N S}^{\gamma}\left(x, Q^{2}, P^{2}\right)$, we find that when we take into account the charge factors, it falls on the singlet quark distribution in almost all $x$ region; namely two "normalized" distributions $\Delta \widetilde{q}_{S}^{\gamma} \equiv \Delta q_{S}^{\gamma} /<e^{2}>$ and $\Delta \widetilde{q}_{N S}^{\gamma} \equiv \Delta q_{N S}^{\gamma} /\left(<e^{4}>-<e^{2}>^{2}\right)$ mostly overlap except at very small $x$ region. The situation is the same in all four factorization schemes. This is attributable to the fact that once the charge factors are taken into account, the evolution equations for both $\Delta \widetilde{q}_{S}^{\gamma}$ and $\Delta \widetilde{q}_{N S}^{\gamma}$ have the same inhomogeneous LO and NLO $\Delta K$ terms and the same initial conditions at $Q^{2}=P^{2}$.

In Fig. 3 we plot the gluon distribution $\Delta G^{\gamma}\left(x, Q^{2}, P^{2}\right)$ beyond the LO in units of $\left(3 N_{f}<e^{2}>\alpha / \pi\right) \ln \left(Q^{2} / P^{2}\right)$, with three different $Q^{2}$ values. Note that it is factorization scheme-independent up to the NLO. We do not see much difference in three lines with different $Q^{2}$. This means the $\Delta G^{\gamma}$ is approximately proportional to $\ln \left(Q^{2} / P^{2}\right)$, or $1 / \alpha_{s}\left(Q^{2}\right)$. But, compared with quark distributions, $\Delta G^{\gamma}$ is very much small in absolute value except at the small $x$ region.

In summary, we have studied the factorization scheme dependence of the parton distributions inside the virtual photon. The scheme dependence is clearly seen in the $n=1$ moments and the large $x$-behaviors of the quark distributions. More details,

together with the analysis on the scheme dependence of parton distributions near $x=0$, will be reported elsewhere.

\section{Acknowledgement}

We thank S. J. Brodsky, J. Kodaira, M. Stratmann and O. V. Teryaev for valuable discussions. This work is partially supported by the Monbusho Grant-in-Aid for Scientific Research NO.(C)(2)-09640345. 


\section{References}

[1] SMC, B. Adeva et al., Phys. Rev. D58 (1998) 112001;

SLAC/E143 Collaboration, K. Abe et al., Phys. Rev. D58 (1998) 112003;

HERMES Collaboration, A. Airapetian et al., Phys. Lett. B442 (1998) 484;

SLAC/E155 Collaboration, P. L. Anthony et al., Phys. Lett. B463 (1999) 339.

[2] M. Glück, E. Reya, M. Stratmann and W. Vogelsang, Phys. Rev. D53 (1996) 475

T. Gehrmann and W. J. Stirling, Phys. Rev. D53 (1996) 6100;

D. de Florian, O. A. Sampayo and R. Sassot, Phys. Rev. D57 (1998) 5803.

[3] G. Altarelli, R. D. Ball, S. Forte and G. Ridolfi, Nucl. Phys B496 (1997) 337;

Acta Phys. Pol. B29 (1998) 1145.

[4] E. Leader, A. V. Sidrov and D. B. Stamenov, Phys. Rev. D58 (1998) 114028;

Phys. Lett. B445 (1998) 232; B462 (1999) 189.

[5] SMC, B. Adeva et al., Phys. Rev. D58 (1998) 112002;

HERMES Collaboration, K. Ackerstaff et al., Phys. Lett. B464 (1999) 123.

[6] G. Altarelli and G. G. Ross, Phys. Lett. B212 (1988) 391;

R. D. Carlitz, J. C. Collins and A. H. Mueller, Phys. Lett. B214 (1988) 229;

V. Efremov and O. V. Teryaev, JINR Report NO. E2-88-287, Dubna, 1988.

[7] G. T. Bodwin and J. Qiu, Phys. Rev. D41 (1990) 2755.

[8] J. Kodaira, Nucl. Phys. B165 (1980) 129.

[9] A. V. Efremov and O. V. Teryaev, Phys. Lett. B240 (1990) 200.

[10] A. Freund and L. M. Sehgal, Phys. Lett. B341 (1994) 90.

[11] S. D. Bass, S. J. Brodsky and I. Schmidt, Phys. Lett. B437 (1998) 424.

[12] M. Stratmann and W. Vogelsang, Phys. Lett. B386 (1996) 370.

[13] K. Sasaki, Phys. Rev. D22 (1980) 2143. 
[14] K. Sasaki and T. Uematsu, Phys. Rev. D59 (1999) 114011; Nucl. Phys. B (Proc. Suppl.) 79 (1999) 614.

[15] T. Uematsu and T. F. Walsh, Phys. Lett.101B (1981) 263.

[16] T. Uematsu and T. F. Walsh, Nucl. Phys. B199 (1982) 93.

[17] H.-Y. Cheng, Int. J. Mod. Phys. A11 (1996) 5109; Phys. Lett. B427 (1998) 371.

[18] D. Müller and O. V. Teryaev, Phys. Rev. D56 (1997) 2607.

[19] R. D. Ball, S. Forte and G. Ridolfi, Phys. Lett. B378 (1996) 255.

[20] W. Furmanski and R. Petronzio, Z. Phys. C11 (1982) 293.

[21] M. Glück and E. Reya, Phys. Rev. D28 (1983) 2749.

[22] M. Glück, E. Reya and A .Vogt, Phys. Rev. D45 (1992) 3986.

[23] M. Fontannaz and E. Pilon, Phys. Rev. D45 (1992) 382.

[24] K. Sasaki and T. Uematsu, in preparation.

[25] J. Kodaira, S. Matsuda, T. Muta, K. Sasaki and T. Uematsu, Phys. Rev. D20 (1979) 627.

[26] R. Mertig and W. L. van Neerven, Z. Phys. C70 (1996) 637.

[27] W. Vogelsang, Phys. Rev. D54 (1996) 2023; Nucl. Phys. B475 (1996) 47.

[28] J. Kodaira, S. Matsuda, K. Sasaki and T. Uematsu, Nucl. Phys. B159 (1979) 99.

[29] S. Narison, G. M. Shore and G. Veneziano, Nucl. Phys. B391 (1993) 69;

G. M. Shore and G. Veneziano, Mod. Phys. Lett. A8 (1993) 373;

G. M. Shore and G. Veneziano, Nucl. Phys. B381 (1992) 23.

[30] K. Sasaki, Prog. Theor. Phys. Suppl. 77 (1983) 197.

[31] W. A. Bardeen and A. J. Buras, Phys. Rev. D20 (1979) 166. 


\section{Figure Captions}

Fig. 1 Factorization scheme dependence of the polarized singlet quark distribution $\Delta q_{S}^{\gamma}\left(x, Q^{2}, P^{2}\right)$ to the NLO in units of $\left(3 N_{f}<e^{2}>\alpha / \pi\right) \ln \left(Q^{2} / P^{2}\right)$ with $N_{f}=3, Q^{2}=30 \mathrm{GeV}^{2}, P^{2}=1 \mathrm{GeV}^{2}$, and $\Lambda=0.2 \mathrm{GeV}$, for $\overline{\mathrm{MS}}$ (dashdotted line), CI (solid line), AB (short-dashed line), and OS (long-dashed line) schemes.

Fig. 2 The polarized singlet quark distribution $\Delta q_{S}^{\gamma}\left(x, Q^{2}, P^{2}\right)$ to the NLO in the OS scheme in units of $\left(3 N_{f}<e^{2}>\alpha / \pi\right) \ln \left(Q^{2} / P^{2}\right)$ with three different $Q^{2}$ values, for $N_{f}=3, P^{2}=1 \mathrm{GeV}^{2}$, and $\Lambda=0.2 \mathrm{GeV}$.

Fig. 3 The polarized gluon distribution $\Delta G^{\gamma}\left(x, Q^{2}, P^{2}\right)$ beyond the LO in units of $\left(3 N_{f}<e^{2}>\alpha / \pi\right) \ln \left(Q^{2} / P^{2}\right)$ with three different $Q^{2}$ values, for $N_{f}=3$, $P^{2}=1 \mathrm{GeV}^{2}$, and $\Lambda=0.2 \mathrm{GeV}$. 


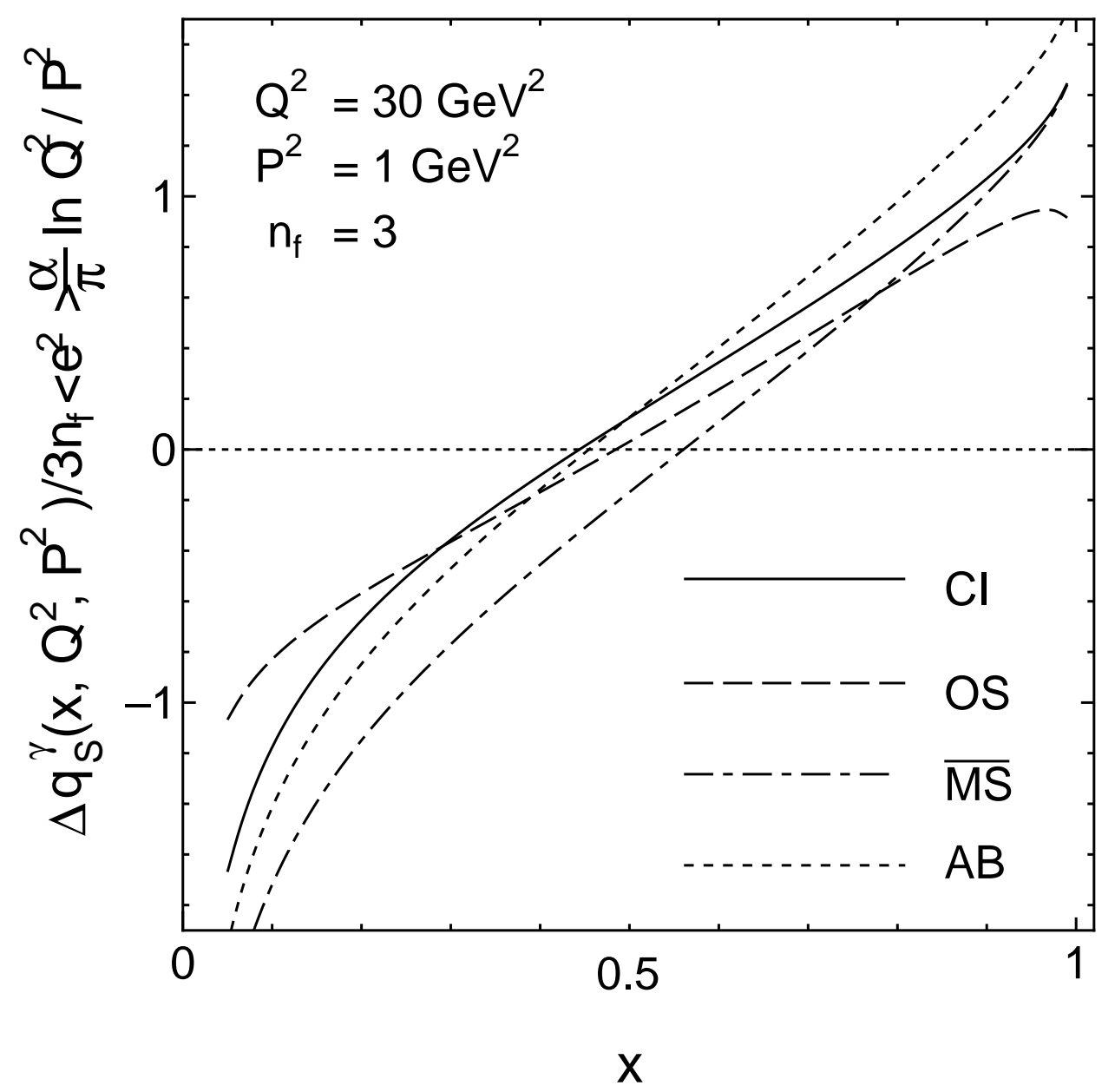

Fig. 1 


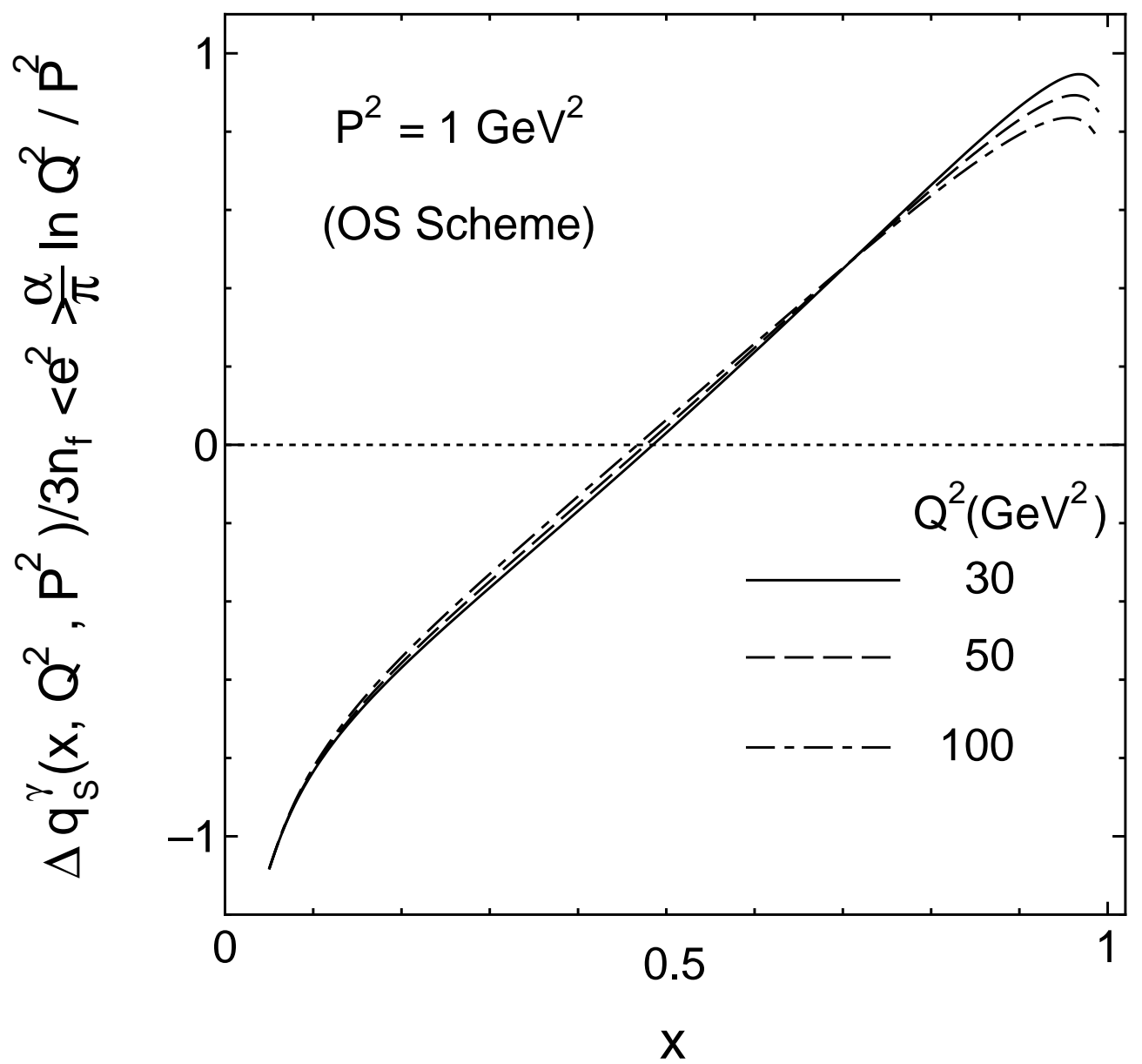

Fig. 2 


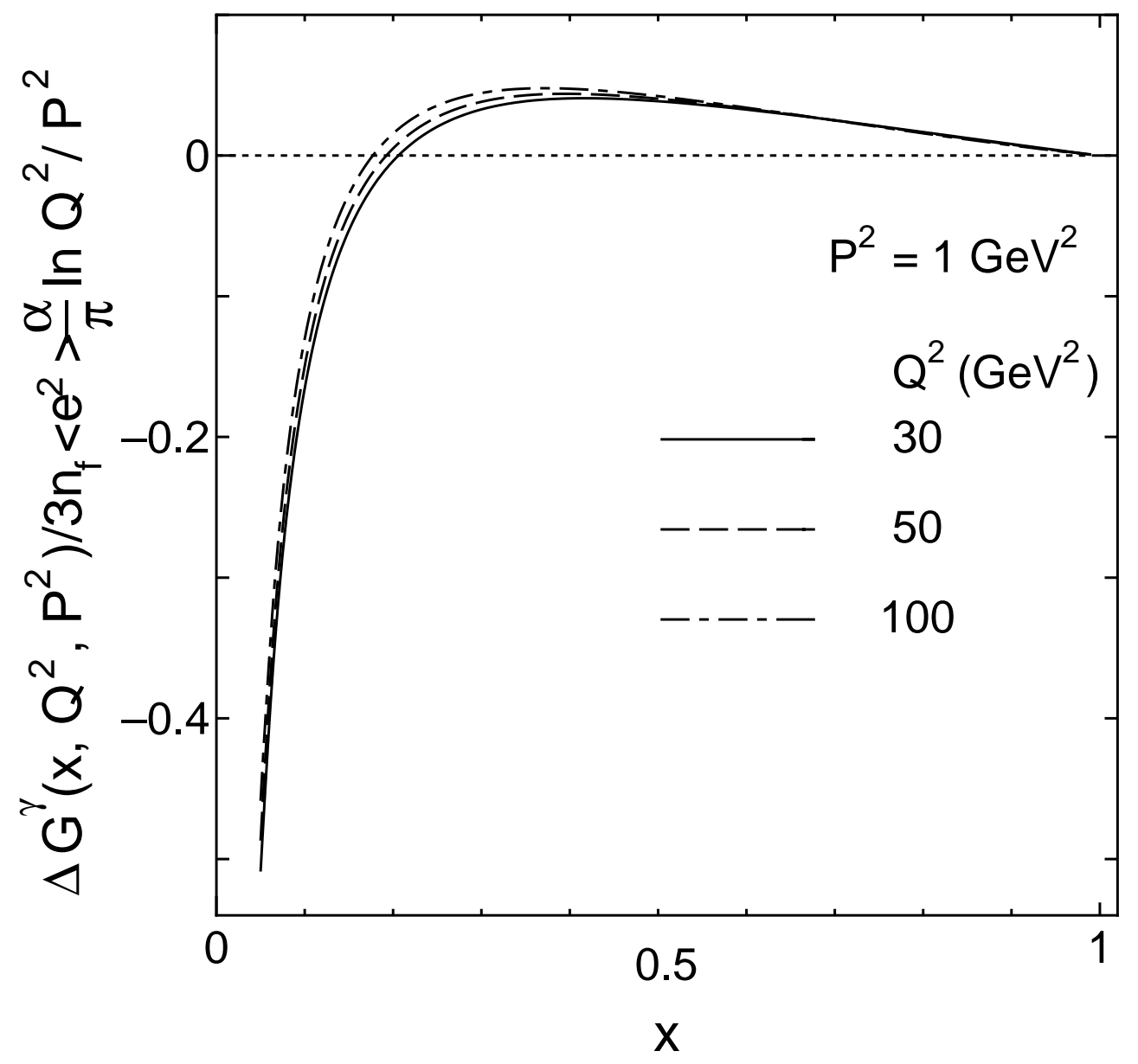

Fig. 3 